\title{
NOVICE DRIVER TRAINING RESULTS AND EXPERIENCE WITH A PC BASED SIMULATOR
}

\author{
R. Wade Allen, George Park, Marcia Cook, Theodore J. Rosenthal \\ Systems Technology, Inc. \\ Hawthorne, CA 90254, USAl \\ E-mail: rwallen@systemstech.com
}

Dary Fiorentino

Southern California Research Institute

Los Angeles, CA 90066

E-mail: dary@adelphia.net

\author{
Erik Viirre \\ University of California at San Diego \\ La Jolla, CA \\ E-mail: eviirre@popmail.ucsd.edu
}

\begin{abstract}
Summary: This paper reports on work accomplished subsequent to a pilot study that was presented at the 2001 conference. This current study will eventually involve the training of over 500 novice drivers, and subsequent comparison of real-world accident and violation rates of the simulator trained group with a traditionally trained control group of demographically matched novice drivers. This paper describes the simulator training system and presents some training data for 111 student subjects collected at three sites involving different simulator configurations. These configurations include a desktop system with a single monitor narrow field of view display, a desktop system with wide field of view display and a cab with wide field of view display. The results include performance measures, a measure of simulator sickness and experience involved in implementing driver-training simulators in the high school environment.
\end{abstract}

\section{INTRODUCTION}

This current work is designed as a transfer of training study and will eventually involve the training of over 500 subjects. Real-world driving accident and violation rates of the simulator trained group will eventually be compared with a demographically matched population of drivers trained by traditional methods to determine if simulator training can improve the safety of accident prone young drivers. Traditional driver's education involves instruction on the driving environment (e.g., rules of the road, traffic control devices, driving hazards) and behind-thewheel training. However, for reasons of safety, the traditional approach does not place novice drivers at risk in real hazardous situations. The premise behind interactive simulator training is that novice drivers can be given experience in dealing with hazardous, cognitively complex driving scenarios that will train their ability in situation awareness, risk assessment and decision making in critical situations. Young drivers traditionally gain this experience in the first several years of driving, but experience high accident rates in doing so. The simulator experience is intended to provide concentrated training in dealing with hazardous situations that young drivers will encounter in the real world after receiving their license. 


\section{BACKGROUND}

The PC-based training system employed in this research has been described in some detail elsewhere (Allen, Rosenthal, et al., 2002). The training system was designed to permit teaching psychomotor and cognitive skills to novice drivers and to be administered by driver education instructors with no prior background in running human subject experiments. The driving scenarios are designed to train critical driving skills, including situation awareness, hazard perception, risk assessment, and decision making under time pressure. Results of a pilot study on PC-based simulator training have previously been presented at this conference (Allen, Cook, et al., 2001), where the performance of novice drivers was compared with that of experienced drivers. In this previous work novice driver performance was shown to approach the level of experienced drivers after six trials performed in blocks of three trials in each of two sessions. Performance results with the current training population have recently been published showing that by the sixth training trial, performance in terms of total accidents and road edge incursions is improved (Allen, Rosenthal, et al., 2003).

\section{TRAINING SYSTEM}

The training system includes features for subject registration, orientation and familiarization, automated administration of simulator runs, data logging, and performance assessment and feedback to the subject. The system consists of a software shell that administers a simple database and can launch applications such as MS Power Point ${ }^{\circledR}$ for orientation briefings, and administer the driving simulator for familiarization and training runs. The software platform also allows simple text-based introductions and summary statements to be placed before and after the administration of each component. The platform elements are as follows:

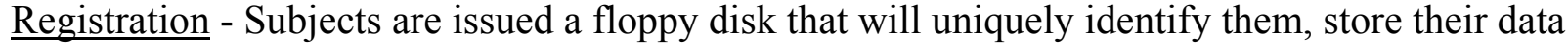
and track their progress. New subjects are asked to enter their name and a unique ID (identification) number. The platform then advances the subject to the orientation.

Orientation - The training system administers a narrated MS Power Point ${ }^{\circledR}$ orientation that provides information and instructions to subjects. The orientation starts with background information to motivate the subject, and then presents important information on the roadway environment and important driving tips such as turning safety and situation awareness concepts including use of rear view mirrors. The orientation ends with a description of the simulator configuration that the subjects will be experiencing, including an overview of the displays and controls. Subjects are assumed to be involved in a more broadly based driver's education curriculum, and the computer orientation is designed to give the information essential for understanding and coping with the roadway environment that will be presented subsequently in the driving simulator training.

Driving Simulation - After the orientation, the training system automatically launches the driving simulator. The first exposure is a familiarization run which slowly introduces the subject to steering and speed control, then intersections with traffic control devices, then finally traffic and pedestrian conflicts. After the familiarization run, the training system presents the subject with standardized training scenarios. The subject is presented with a number of driving scenarios during which performance is evaluated. Subjects can 'graduate' on the sixth trial if they meet performance criteria including no accidents, no more than one ticket, and reasonable use of the turning indicators. If subjects do not graduate on the sixth trial, they are administered up to three 
more trials to achieve the graduation criteria. If they do not 'graduate' on the ninth trial the training system thanks them for their participation and admonishes them to drive carefully and practice the safe driving tips presented by the training system.

\section{TRAINING SYSTEM PERFORMANCE}

The training system has been deployed in three configurations as illustrated in Figure 1. A simple single-monitor version has been deployed in three high schools. The three-monitor, wide fieldof-view desktop system and the cab system with a curved projection screen have been deployed in research laboratories. The school systems have been administered by teachers, and students in driver's education classes have been assigned to complete the training regimen as a class requirement. The research laboratories have recruited teenagers applying for learner's permits at California State Department of Motor Vehicles field offices. Data reported herein was collected on the three configurations illustrated in Figure 1. Performance and simulator sickness data were analyzed with MANOV methods according to a mixed effect design, with simulator Configuration and Gender as between-subject variables, and Trials as a within-subject variable. Performance measures included accidents, speeding, road edge incursions and time-to-collision. Multivariate tests showed statistical significance for Configuration $(\mathrm{p}<.001)$, Gender $(\mathrm{p}=.012)$, and Trials $(p<.001)$. Significant interactions included Trials $x$ Configuration $(p=.03)$ and Trials $x$ Configuration $\mathrm{x}$ Gender $(\mathrm{p}<.015)$
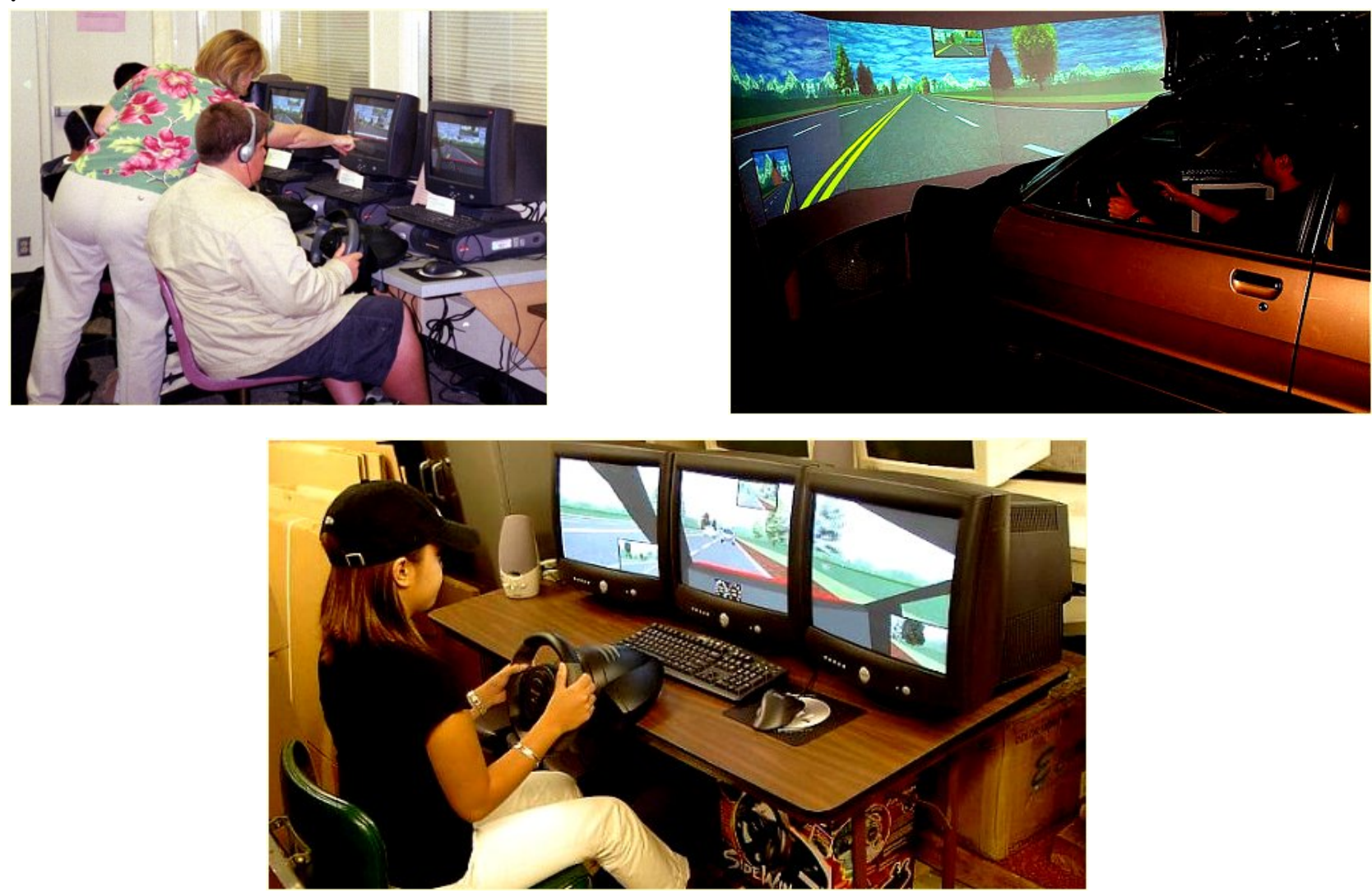

Figure 1. Novice Driver Simulator Configurations 
Performance results on accidents and road edge incursions have been reported previously for the three configurations shown in Figure 1 (Allen, Cook, et al., 2001). Figure 2 shows results for Speed Limit Exceedances with the three simulator configurations for the first six training trials. The configuration and trial effects and the interaction are statistically significant $(p=0.01, p=.005$ and $\mathrm{p}=0.05$, respectively). There is a general improvement from the first to the sixth trial, but there are clearly some differing effects between the configurations deployed at different sites. The single monitor desktop configuration, which is associated with the poorest performance, was deployed at a high school. The single monitor gives the least amount of peripheral speed cues, which may explain this result.

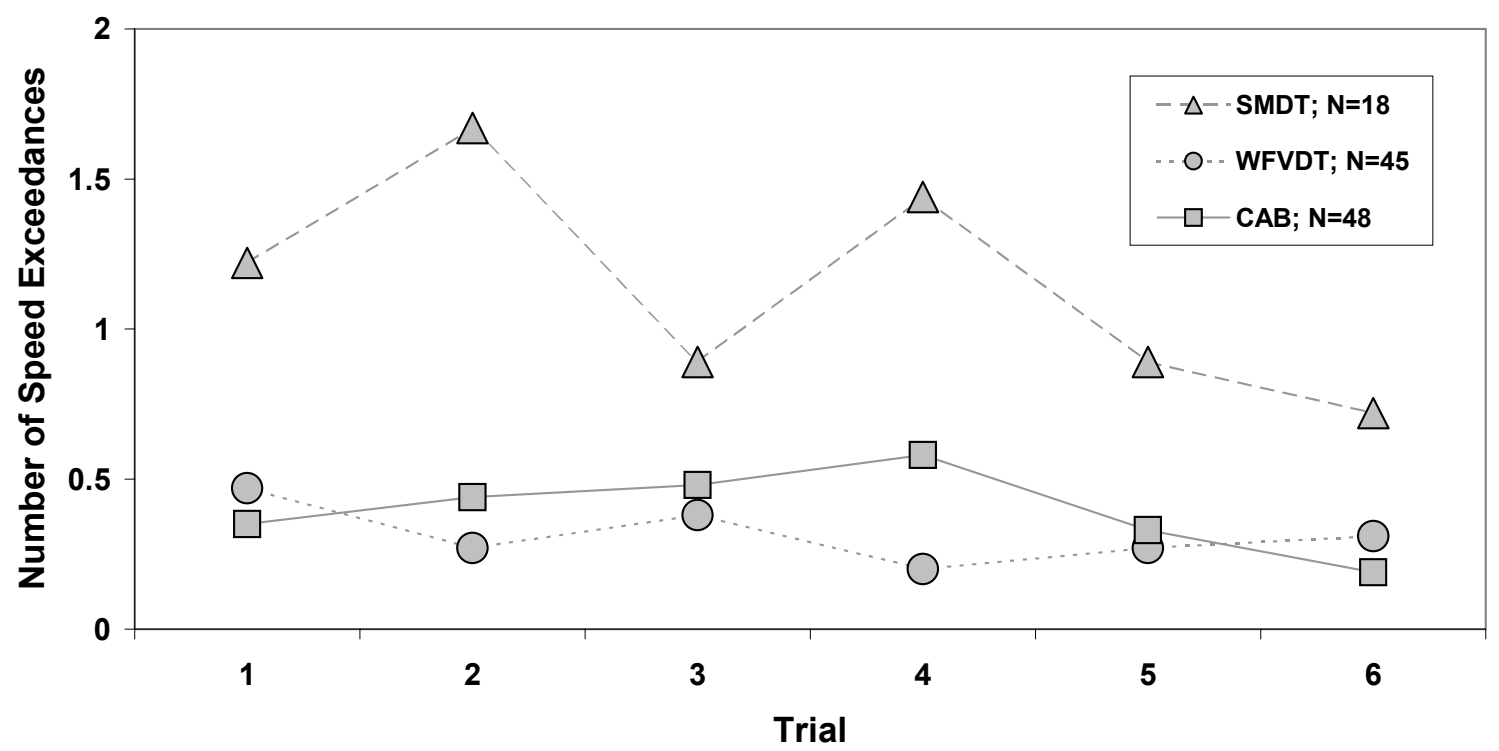

\section{Figure 2. Number of Speed Limit Exceedances for Three Simulator Configurations: SMDT - Single Monitor Desk Top; WFVDT - Wide Field of View Desk Top; CAB - Cab with Wide Field of View Projection}

At the end of each training trial students were asked to rate whether they were experiencing any discomfort on a four point scale: $0=$ fine; $1=$ a little queasy; $2=$ moderate, but can continue; $3=$ would like to stop. Out of a total of 999 responses, $726(91.7 \%)$ were 'fine,' $22(2.8 \%)$ were 'a little queasy,' $25(3.2 \%)$ were 'moderate but could continue,' and $19(2.4 \%)$ indicated they 'would like to stop.' Figure 3 shows results of the average simulator sickness scores over trials by gender. The Gender effect was statistically significant $(\mathrm{p}=.005)$, which is consistent with past research (e.g., Kolasinski, 1995) while Trials was not ( $\mathrm{p}>.10)$, which indicates minimal habituation to the environment. Simulator configuration was also not significant. Typically simulator sickness symptoms are considered to increase with display field of view (e.g., Kolasinski, 1995), but the data here does not demonstrate that effect.

Regarding operational considerations, the training system has proven to be relatively robust in its deployment at the high schools where it is administered by staff personnel. A major goal of this project was to set up a system that could be deployed outside of the laboratory environment in a typical high school driver's education program. The training system configuration was designed to be administered by non-research personnel in collaboration with driver education teachers. 
We were successful in adapting high school media lab computers to run the single monitor desktop training system, which represents a major cost consideration for future deployment of computer-based driver training systems.

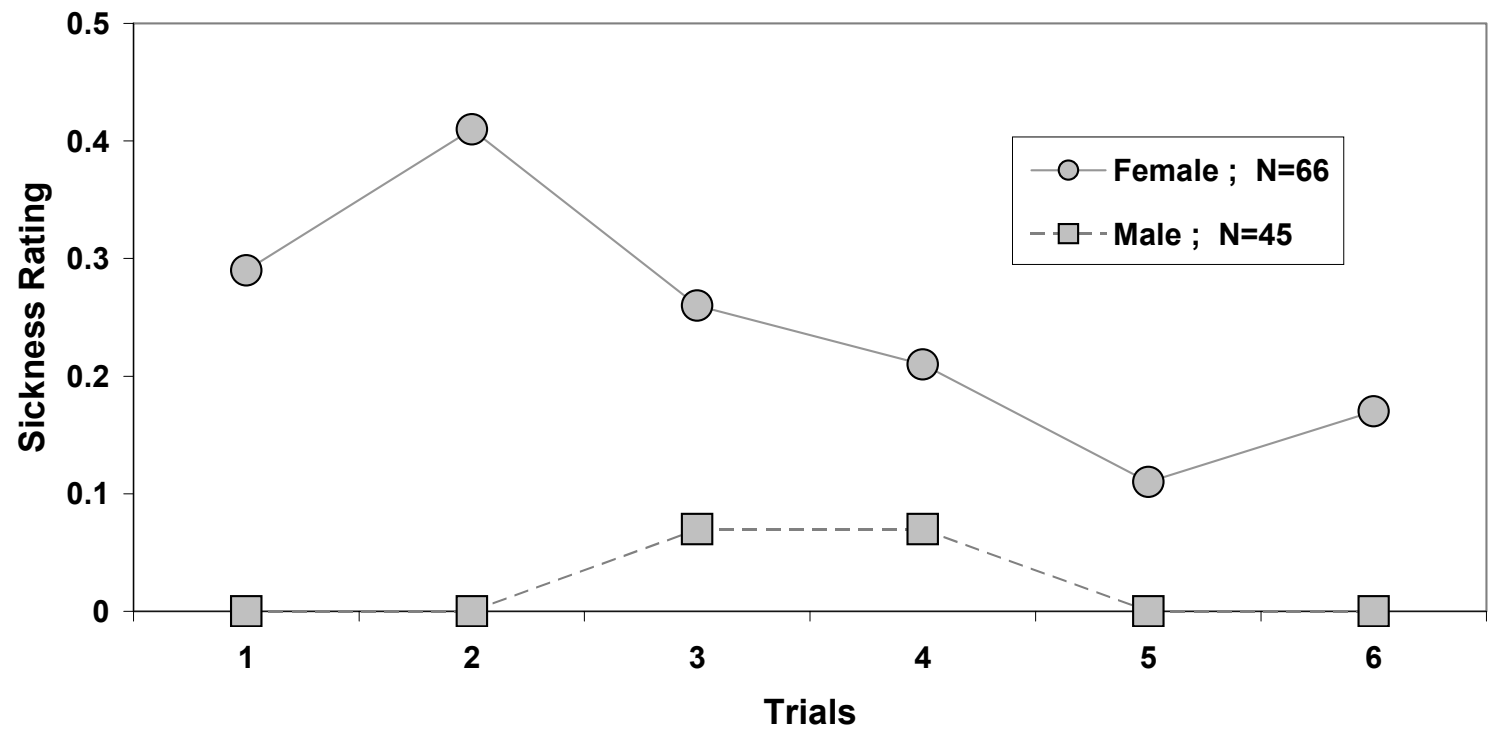

Figure 3. Simulator Sickness Rating for Females and Males

\section{CONCLUDING REMARKS}

The objective of this research was to develop and demonstrate a PC-based driver training system that can be routinely run outside of the research laboratory by non-research personnel (e.g., trainers, teachers). The driving scenarios and performance measures have proven to be sensitive to training. The PC-based platform described herein has proven to be easy to administer in school classrooms as well as in laboratories. PC technology will continue to improve for the foreseeable future in terms of computing power and visual and auditory display. The potential capability of PC-based training and evaluation platforms will also advance, limited primarily by software development. The long-range goal of this transfer-of-training project still remains to be assessed. This will be considered in the next few years, when adequate accident and violation incidences have accumulated in the California Department of Motor Vehicles database.

\section{REFERENCES}

Allen, R.W., Cook, M.L., et al. (2001). Low Cost PC Simulation Technology Applied to Novice Driver Training. Proceedings of the International Driving Symposium on Human Factors in Driver Assessment, Training, and Vehicle Design. Iowa City, IA: The University of Iowa.

Allen, R.W., Rosenthal, T.J., et al. (1999). Low Cost, PC-Based Techniques For Driving Simulation Implementation. DSC1999, Paris, France.

Allen, R.W., Rosenthal, T.J., et al. (2003). Assessment and Training Using a Low Cost Driving Simulator. 5th International Conference on Engineering Psychology and Cognitive Ergonomics, Crete, Greece, June. 
Allen, R.W., Rosenthal, T.J., et al. (2002). A Low Cost Platform for Training and Evaluating Driver Behavior. DSC2002, Paris, France, September.

Kolasinski, E.M. (1995). Simulator Sickness in Virtual Environments. U.S. Army Research Institute for the Behavioral and Social Sciences, Alexandria, VA, Final ARI TR 1027. 